\title{
Geometric Comments on Music
}

\author{
VADOUD NAJJARI \\ Department of mathematics, Islamic Azad university, Maragheh branch, \\ Maragheh, IRAN
}

Abstract: In fact degrees of the middle chromatic scales are related with the logarithm (base 2). With relying on this fact, In this study by using logarithm and string oscillation properties, we show that music notes $(\mathrm{A}-\mathrm{B}-\cdots)$ are related with the interval $(0, \mathrm{a}]$ ( $\mathrm{a}$ is constant) and then we define a topology on this interval. Finally Lie algebra for the mentioned interval of music will be characterized.

KeyWords: Lie group, Lie algebra, Music notes, String oscillation, Topology.

Received: May 16, 2021. Revised: September 28, 2021. Accepted: October 7, 2021. Published: October 21, 2021.

\section{Introduction}

Music is one of the interesting phenomenons of the nature for everybody. Different persons like deferent types of the music and they have deferent perception of them. There are some people that like to analyze music with different point of view, like as, Mathematically, probabilistic, algebraic, wavelets, physical and etc (see [4], [7], [8], [9], [13], [14], [15], [16]). Temperley is one of the popular persons that has very interesting idea in music theory and has published a lot of papers in this subject. Also he has introduced a new probabilistic point of view in his book [13]. He has proposed a new Bayesian method to musical modeling, although he was not the first one (see [10], [11], [12], [17]). Namely he has a general study of probabilistic modeling of the music is his book. More extensive treatments can be found elsewhere. Coverage of basic probability and Bayes rule can be found in probability texts such as Safran et al. (1999) and Ross (2000).

In this study we try to look at music with topological point of view that has not been seen before.

The rest of this paper constructed as follows. In section 2 we see some useful preliminaries. Section 3, reviews some geometric topics such as Lie group and Lie Algebra. Section 4 , includes logarithmic representation of music. And section 5 is related with music and Lie algebra.

\section{Preliminaries}

We are able to hear noises that their frequency are between $C$ note 16 hertz (one octave flater than the flattest $C$ note in the piano) and the flat $E$ note (near 2000 hertz)(see [9], [12]). Now we review some basic definitions in theory of music, for details you can look [9], [12].

Definition 2.1: Interval in the Music is difference of sharp and flat or ordering ratio between two sounds. Interval of the two successive notes is called connected and others is called detached. Some of connected intervals, that are closer to each other are said half tone and others are called major tone.

Definition 2.2: Frequency ratio between $C$ note and subsequent $C$ note is $\frac{1}{2}$. This ratio is called Octave.

Example 2.3: $C_{3}=264$ ( $C$ note in the third octave) and $C_{4}=522(C$ note in the fourth octave $)$
Definition 2.4: Two notes that haven't same name and their between interval is half tone, will be called diatonic half tone. Two notes that have same name and their between interval is half tone, will be called chromatic half tone.

Definition 2.5: Scale is the sounds or notes that lay continuously between octave. In the other means, scale is the regular rows of sounds.

Definition 2.6: Diatonic scale is composed with diatonic tones and diatonic half tones. Chromatic scale is composed from 12 half tones ( 7 diatonic half tones and 5 chromatic half tones).

As electronic musicians, we deal with all sorts of numbers whose resolution changes drastically from low to high. Consider, for example, the relatively simple and familiar concept of musical octaves. Because an increase in pitch of one octave corresponds to a doubling of the fundamental frequency, an octave above $20 \mathrm{~Hz}$ is $40 \mathrm{~Hz}$, whereas an octave above 1,000 $\mathrm{Hz}$ is $2,000 \mathrm{~Hz}$. Between 20 and $40 \mathrm{~Hz}$, a variation of $1 \mathrm{~Hz}$ represents 5 percent $(1 / 20)$ of the octave - between 1,000 and $2,000 \mathrm{~Hz}$, it represents only 0.1 percent $(1 / 1,000)$ of the octave.

To put this doubling in mathematical terms, as the octave increases, frequency increases by a power of two: the frequency $n$ octaves above a pitch $P$ is $P \times 2^{n}$. Because $n$ is literally the exponent of 2 , this is called an exponential relationship. If pitch rises at a steady rate, as when a musician plays an ascending chromatic scale, frequency rises at an increasing rate. The distance in terms of frequency between each subsequent pair of notes is greater than the distance between the previous pair of notes, yet musically each is a semitone (half step).

A logarithm is the power to which a certain base $b$ must be raised to equal a particular number $x$. In other words, if $b^{n}=x$, then $\log _{b}(x)=n$. Logarithms solve two problems. First, they allow us to make meaningful comparisons of things, such as musical intervals, when the underlying numeric relationships vary according to range. An octave is still an octave whether it spans $20 \mathrm{~Hz}$ or 1,000 Hz. Second, logarithms 
enable us to describe very large and very small numbers with relatively simple numbers, as you'll see.

Our ears can detect the sound of an insect's wings at arm's length, and our ears can be instantly damaged by a train horn at the same distance. In between are all the useful volumes. The ratio between the sound pressure of these two sounds is about a million to one. Imagine a manufacturer describing the signal-to-noise ratio of a new preamble in those terms. Of course, this is why we use decibels (see [5], [6], [8], [9], [12], [18]. Decibels define the ratio between two powers, so we can make meaningful comparisons between two numbers regardless of whether we're talking pascals or micropascals. They also narrow that million-to-one span to a range of 120 $\mathrm{dB}$. A bel is the power to which 10 must be raised to equal the ratio in question - by definition, it's a logarithm. A decibel is simply a tenth of a bel. If sound A is 100 times louder than sound $\mathrm{B}$, the ratio of their intensities is $100: 1$, or 100 . Because $100=10^{2}$, the ratio is 2 bels, or 20 decibels.

Mathematically, $d=10 \log _{10}\left(I_{A} / I_{B}\right)$, with $d$ representing the number of decibels and $I_{A}$ and $I_{B}$ representing the intensities of sounds $A$ and $B$, respectively. The decibel, being a logarithm, allows us to describe the relative power of two signals regardless of whether their absolute powers are very small numbers or very large numbers. The numbers tend to get a bit squirrelly when decibels are used to describe different things. If you double the power of a signal, it increases by $3 \mathrm{~dB}$ : because $10^{0.3}=2$ (approximately), $10 \log _{10}(2 / 1)=10 \times 0.3=3$. However, since power increases by the square of the voltage, doubling the voltage yields an increase of $6 \mathrm{~dB}$. The math for this depends on the simple fact that squaring a number multiplies its logarithm by 2 . We therefore use $20 \log _{10}(2 / 1)=20 \times 0.3=6$. To double the subjective loudness of a sound requires about ten times the power, so "twice as loud" means $10 \mathrm{~dB}$ higher: $10 \log _{10}(10 / 1)=10 \times 1=10$. If all the math just makes you reach for the aspirin, just remember that a decibel is a logarithmic unit that enables us to cover a million-to-one scale with ease and clarity.

\section{Lie Group and Lie Algebra}

In this section we review some geometric topics such as Lie group and Lie Algebra, for details you can see [2], [3]. We assume that reader is familiar with geometry.

Definition 3.1: A analytic manifold $G$ is a Lie group, if $G$ be a group and $\varphi$ be analytic map:

$$
\begin{gathered}
\varphi: G \times G \longrightarrow G \\
(x, y) \longrightarrow x \cdot y^{-1}
\end{gathered}
$$

Example 3.2: $R^{*}=R-\{0\}$ is a Lie group.

Now we review a theorem that will be useful for our aim in the next section. For proof and details of this theorem you can see [3].

Theorem 3.3: Let $G$ be a Lie group and $H$ be a closed subgroup of $G$ then $H$ is a sub Lie group of $G$.
We shall say that a vector space $\bar{g}$ over $\mathbb{R}$ is a (real) Lie algebra if (in addition to its vector space structure) it possesses a product, that is a map

$$
\begin{gathered}
\Phi: \bar{g} \times \bar{g} \longrightarrow \bar{g} \\
(x, y) \longrightarrow[x, y]
\end{gathered}
$$

which has the following properties:

1) It is bilinear over $\mathbb{R}$,

$$
\begin{aligned}
{\left[a x_{1}+b x_{2}, y\right] } & =a\left[x_{1}, y\right]+b\left[x_{2}, y\right] \\
\left.x, a y_{1}+b y_{2}\right] & =a\left[x, y_{1}\right]+b\left[x, y_{2}\right]
\end{aligned}
$$

2) It is skew commutative,

$$
[x, y]=-[y, x]
$$

3) It satisfies the Jacobi identity,

$$
[x,[y, z]]+[y,[z, x]]+[z,[x, y]]=0
$$

Theorem 3.4: There are open neighborhoods $u_{0}$ of 0 in $\bar{g}$ and $v_{e}$ of $e$ in $G$ such that the following exponential map is a diffeomorphism.

$$
\exp : u_{0} \longrightarrow v_{e}
$$

See [3].

Proposition 3.5: Let $G=\mathbb{R}^{n}$ then we will have

$$
T_{x} \mathbb{R}^{n}=\{x\} \times \mathbb{R}^{n}=\mathbb{R}^{n}
$$

by defining $l_{a}(x)=a+x$ we have

$$
\begin{gathered}
d l_{a}:\{x\} \times R^{n} \longrightarrow\{a+x\} \times R^{n} \\
(x, u) \longrightarrow(x+a, u)
\end{gathered}
$$

for $A \in T_{0} \mathbb{R}^{n}$ we consider vector field $\bar{A}$ in $\mathbb{R}^{n}$ that is created with translation, $\bar{A}(x)=d l_{x}(A)$. If

$$
A=\sum a^{i}\left(\frac{\partial}{\partial x^{i}}\right)
$$

we have,

$$
\bar{A}(x)=\sum a^{i}\left(\frac{\partial}{\partial x^{i}}\right)_{x}
$$

therefore $\bar{A}$ is a vector field with constant coefficient, now if $\bar{A}, \bar{B}$ be vector fields, then $[\bar{A}, \bar{B}]=0$ and Lie algebra of $\bar{g}$ will be $\mathbb{R}^{n}$.

Corollary 3.6: For all $a \in \mathbb{R}^{*}$, we have $T_{a} \mathbb{R}^{*}=\mathbb{R}$.

\section{Logarithmic Representation of Music}

In fact degrees of the middle chromatic scales is related with logarithm (base 2). Assume that $C$ note, in the lowest octave -that we say zero octave- do $n$ oscillation per second. Hence $C$ note in the first octave will do $2 n$ oscillation and in octave $m$, it will do $n \times 2^{m}$ oscillation and so on.

Let $P$ be all chromatic notes, we assume that the main tone $C$ in any octave is zero, therefore for example $G$ tone will be the seventh and $A$ tone will be the ninth and so on, the twelve tone again will be $C$ with higher octave. In every middle chromatic scales, every tone oscillation is equal to number 
of previous oscillation tone (multiplication with $\sqrt[12]{2}$ ). Hence for every tone oscillation we have

$$
\begin{aligned}
N_{p m} & =n \times 2^{m}(\sqrt[12]{2})^{p} \\
\log N_{p m} & =\log n+m \log 2+P \frac{\log 2}{12} \\
\log N_{p m} & =\log n+\left(m+\frac{p}{12}\right) \log 2
\end{aligned}
$$

if we assume that, the oscillation of the lowest $C$ note is $1,(n=1)$ and logarithm base is 2 then

$$
\log _{2} N_{p m}=m+\frac{p}{12}
$$

that shows piano key numbers are the logarithm of the oscillation of related sounds (multiplication 12), and octave number is the characteristic of the logarithm and also sound number in the related octave (divide 12) is the logarithm mantissa. For example tone of $C$ note, in the third octave is $3+7 / 12$, that 3 describes the oscillation number and $7 / 12(=0.583)$ is the mantissa, therefore oscillation will be $2^{3.583}=11.98$ times the oscillation of the $C$ note in the first octave.(see also [1], [5], [12], [18].

\section{Music an Lie Algebra}

We know that pitching of the musical instrument, changes frequencies and oscillation of $C$ note in the first octave (and therefore other notes), hence oscillations will be in the interval $[1, a]$, ( $a$ is constant). Now without loss of generality, we assume that oscillations are in the interval $\Omega=(0, a]$.

Lemma 5.1: By defining an action for all $x, y \in \Omega=(0, a]$, $\Omega$ can be an abelian group.

Definition 5.2: Let $\mathbb{R}$ be the real number set, and let $\tau=$ $\{(r,+\infty) \mid r \in \mathbb{R}\} \bigcup\{\mathbb{R}, \emptyset\}$ then $\tau$ is a topology for $\mathbb{R}$. $\tau$ will be called right radius topology.

Lemma 5.3: $\Omega$ is closed in $\mathbb{R}^{*}$.

It is enough to consider $\mathbb{R}^{*}$ with right radius topology.

Corollary 5.4: Theorem 3.3 and lemma 5.3 show that $\Omega$ is a sub Lie group of $\mathbb{R}^{*}$, since for all $a \in \mathbb{R}^{*}, T_{a} \mathbb{R}^{*}=\mathbb{R}$. Also $\ln x=\frac{\log _{2}^{x}}{\log _{2}^{e}}$ and music notes are related with logarithm base 2, therefore we obtain the Lie Algebra of the music interval with multiplication $\log _{2}^{e}$ to any vector in $T \Omega$ (Lie algebra of $\Omega)$.

\section{References}

[1] E. D. Blackhom, The Physics of the Piano, Scientific American December 1965.

[2] T. Brocker and Dieck, Representation of Compact Lie Groups, New Mexico, USA. 1991.

[3] H. D. Fegan, Introduction to Compact Lie Groups, New Mexico, USA. 1991.

[4] M. Ferrand, A. Smaill (eds.), Music and Artificial Intelligence, Springer . Berlin. pp 195-206.

[5] D. Halliday, R. Resnick, Fundamentals of PHYSICS, USA. 1974

[6] M. Hutchins, The Physics of the Violins, Scientific American. November 1962.

[7] D.J. Povel, Exploring the fundamental harmonic forces in the tonal system, Psychological Research. 58 (1996) 274-283.

[8] J. Rahn, Basic Atonal Theory, New York: Schirmer. 1980.

[9] J. L. Snyder, Entropy as a measure of musical style: The influence of a priori assumptions. Music Theory Spectrum. 12 (1990) 121-160.

[10] S. Ross, Introduction to Probability Models. San Diego: Academic Press. 2000.
[11] J. R. Saffran, E. K. Johnson, R. N. Aslin, E. L. Newport, Statistical learning of tone sequences by human infants and adults, Cognition. 70 (1999) 27-52.

[12] C. E. Schachter, Rhythm and linear analysis: A preliminary study. Music Forum. 4 (1976) 281-334.

[13] D. Temperley, Music and probability, Massachusetts Institute of Technology The MIT Press,Cambridge, Massachusetts London, England. 2007.

[14] D. Temperley, A Bayesian approach to key-finding, In C. Anagnostopoulou. 2002.

[15] D. Temperley, Communicative pressure and the evolution of musical styles, Music Perception. 21 (2004) 313-337.

[16] D. Temperley, The question of purpose in music theory: Description, suggestion, and explanation, Current Musicology. 66(2001b) 66-85.

[17] J. B. Tenenbaum, Bayesian modeling of human concept learning, In $M$. S. 1999.

[18] S. Wilkinson, Decibels Demystified, Electronic Musician. 2001.

\section{Creative Commons Attribution License 4.0 (Attribution 4.0 International, CC BY 4.0)}

This article is published under the terms of the Creative Commons Attribution License 4.0 https://creativecommons.org/licenses/by/4.0/deed.en US 